\title{
Some plethysm results related to Foulkes' conjecture
}

\author{
Steven Sivek \\ Department of Mathematics \\ Massachusetts Institute of Technology \\ ssivek@mit .edu
}

Submitted: Aug 17, 2005; Accepted: Mar 7, 2006; Published: Mar 14, 2006

Mathematics Subject Classification: 05E10

\begin{abstract}
We provide several classes of examples to show that Stanley's plethysm conjecture and a reformulation by Pylyavskyy, both concerning the ranks of certain matrices $K^{\lambda}$ associated with Young diagrams $\lambda$, are in general false. We also provide bounds on the rank of $K^{\lambda}$ by which it may be possible to show that the approach of Black and List to Foulkes' conjecture does not work in general. Finally, since Black and List's work concerns $K^{\lambda}$ for rectangular shapes $\lambda$, we suggest a constructive way to prove that $K^{\lambda}$ does not have full rank when $\lambda$ is a large rectangle.
\end{abstract}

\section{Introduction}

Let $\lambda=\left(\lambda_{1}, \lambda_{2}, \ldots, \lambda_{r}\right)$ be the shape of a Young diagram with $|\lambda|=\sum \lambda_{i}$ boxes in rows of length $\lambda_{1} \geq \lambda_{2} \geq \ldots \geq \lambda_{r}$, and define a tableau as a way to fill such a diagram with the numbers $1,2, \ldots,|\lambda|$ in some order. Following the notation in [4], we declare two tableaux to be $h$-equivalent, denoted $\equiv_{h}$, if one can be obtained from the other by permuting elements within a row and swapping rows of equal length, and likewise two tableaux are $v$-equivalent, denoted $\equiv_{v}$, if the same can be done by manipulating columns rather than rows. In other words, two tableaux are $h$-equivalent if they represent the same partition of $\{1,2, \ldots,|\lambda|\}$ with shape $\lambda$ (i.e. in blocks of length $\lambda_{1}, \ldots, \lambda_{r}$ ), and they are $v$-equivalent if they represent the same partition with shape $\lambda^{\prime}$, where $\lambda^{\prime}$ denotes the transpose of $\lambda$.

Denoting by $T^{\lambda}$ the set of all tableaux of shape $\lambda$, we can now define row tabloids to be members of the set of $\equiv_{h^{-}}$equivalence classes $H^{\lambda}:=T^{\lambda} / \equiv_{h}$ and column tabloids to be members of the set $V^{\lambda}:=T^{\lambda} / \equiv_{v}$. We define an orthogonality relation as follows: given $\Gamma \in H^{\lambda}$ and $\Delta \in V^{\lambda}$, we say $\Gamma \perp \Delta$ if for every row $r$ of $\Gamma$ and column $c$ of $\Delta$ we have $|r \cap c| \leq 1$. Equivalently, we first define orthogonality on $T^{\lambda}$ by saying that $\Gamma \perp \Delta$ for $\Gamma, \Delta \in T^{\lambda}$ if there exists some tableau $T$ such that $\Gamma \equiv_{h} T$ and $\Delta \equiv_{v} T$, and then we can extend this naturally to a relation between $H^{\lambda}$ and $V^{\lambda}$. Finally, we construct a matrix 


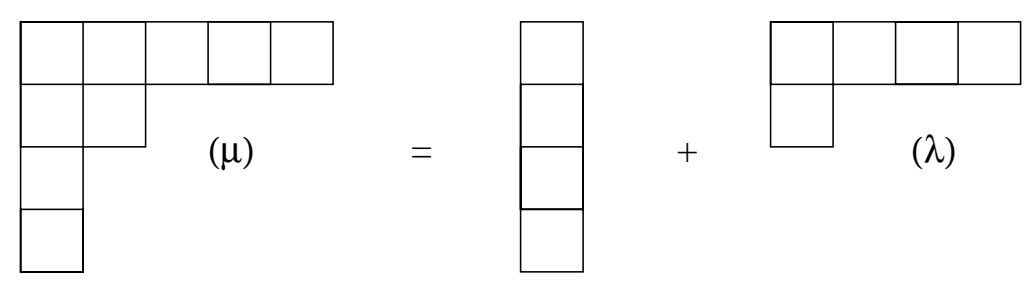

Figure 1: $\mu$ extends $\lambda$, where $\mu=(5,2,1,1), \lambda=(4,1)$, and $\mu-\lambda=(1,1,1,1)$.

$K^{\lambda}$ with $\left|H^{\lambda}\right|$ rows and $\left|V^{\lambda}\right|$ columns, each indexed by the members of their respective sets, such that for $\Gamma \in H^{\lambda}$ and $\Delta \in V^{\lambda}$, the entry $K_{\Gamma, \Delta}^{\lambda}$ is 1 if $\Gamma \perp \Delta$ and 0 otherwise. We may think of $K^{\lambda}$ as a map $\mathbb{Q} V^{\lambda} \rightarrow \mathbb{Q} H^{\lambda}$ of $\operatorname{Sym}(|\lambda|)$-modules, though here it will generally suffice to simply view it as a matrix.

Let $S^{k} V$ denote the $k$ th symmetric power of a finite-dimensional complex vector space $V$. Foulkes' plethysm conjecture states that if $n \geq m$, then the $\operatorname{Sym}(m n)$-module $S^{n}\left(S^{m} V\right)$ contains $S^{m}\left(S^{n} V\right)$ as a submodule. Though the conjecture is still unresolved, Black and List suggested a method of attack in [1] by constructing a particular map $\phi^{m, n}: S^{m}\left(S^{n} V\right) \rightarrow S^{n}\left(S^{m} V\right)$ (or equivalently, $\mathbb{Q} V^{n \times m} \rightarrow \mathbb{Q} H^{n \times m}$ ) given by the matrix $K^{n \times m}$, where the shape " $n \times m$ " is a rectangle with $n$ rows and $m$ columns. They then showed that if this map is injective for $n \geq m>1$, or equivalently if $K^{n \times m}$ has full rank, then $K^{n \times r}$ also has full rank (i.e. $\phi^{r, n}$ is injective) for all pairs $(n, r)$ where $m \geq r \geq 1$, and so Foulkes' conjecture holds for all of these pairs. Stanley generalized this by conjecturing in [5] that if $\lambda$ is a shape such that $\lambda \geq \lambda^{\prime}$ in dominance order, then the rows of $K^{\lambda}$ are linearly independent. Noting that some shapes with this property yield matrices with more rows than columns, Pylyavskyy modified the conjecture in [4] to the following:

Conjecture 1.1. $K^{\lambda}$ has full rank for all $\lambda$.

This conjecture is in general false: Müller and Neunhöffer showed in [2] that $K^{5 \times 5}$ does not have full rank, so not only do Stanley's and Pylyavskyy's conjectures fail but the approach of Black and List does not suffice to prove Foulkes' conjecture.

We will show that Conjecture 1.1 fails for many more cases in the sense that every shape $\lambda$ can be nested inside some $\mu$ for which $K^{\mu}$ does not have full rank, and furthermore that the rank of $K^{\lambda}$ can be arbitrarily small compared to $\min \left(\left|H^{\lambda}\right|,\left|V^{\lambda}\right|\right)$. We will then address the case where $\lambda$ is an $m \times n$ rectangle and consider two ways to prove that such $K^{\lambda}$ are not rank-maximal, first by attempting to find an upper bound on $\operatorname{rank}\left(K^{\lambda}\right)$ and then by conjecturing that $\operatorname{ker}\left(K^{\lambda}\right)$ contains vectors of a particularly convenient form.

\section{Extending shapes}

Let $\lambda$ and $\mu$ be Young diagrams. We will say that $\mu$ extends $\lambda$ if for some $k \geq 1$, we can delete the leftmost $k$ columns from $\mu$ so that the remaining shape is $\lambda$. We will denote the shape of these $k$ columns by $\mu-\lambda$; see Figure 1 for an example. 
Many of our counterexamples to Conjecture 1.1 will be constructed from the following lemma.

Lemma 2.1. Given a shape $\lambda$, if there exists a nonzero vector $v$ with $K^{\lambda} v=0$, then there exists a nonzero vector $w$ such that $K^{\mu} w=0$ for any $\mu$ which extends $\lambda$.

Proof. Recall that the entries of $K^{\lambda}$ are indexed by pairs $(\Gamma, \Delta)$ of row and column tabloids. If $v=\left(v_{\Delta}\right)_{\Delta \in V^{\lambda}}$, then $\sum_{\Delta \in V^{\lambda}} K_{\lambda}^{\Gamma, \Delta} v_{\Delta}=0$ for all row tabloids $\Gamma \in H^{\lambda}$.

Suppose that $\lambda$ has $b$ boxes, $\mu$ has $c>b$ boxes, and $\mu-\lambda$ has $k$ columns; clearly it suffices to consider the case $k=1$. For each $\Delta \in V^{\lambda}$, construct a column tabloid $\Delta^{\prime}$ of shape $\mu$ by filling in the rightmost columns which correspond to the shape $\lambda$ exactly as in $\Delta$, and assigning $b+1, \ldots, c$ to the first column. Finally, construct $w=\left(w_{\delta}\right)_{\delta \in V^{\mu}}$ as follows: set $w_{\Delta^{\prime}}=v_{\Delta}$ for all $\Delta \in V^{\lambda}$, and let all other $w_{\delta}$ be 0 . Note that for any distinct $\Delta_{1}, \Delta_{2} \in V^{\lambda}$, we have $\Delta_{1}^{\prime} \not \equiv_{v} \Delta_{2}^{\prime}$ since $\Delta_{1} \not \equiv_{v} \Delta_{2}$, so this construction is well defined, and that since $v$ is nonzero we have $v_{\Delta} \neq 0$ for some $\Delta$, hence $w$ is nonzero.

We must now show that $K^{\mu} w=0$. Fix an arbitrary row tabloid $\Gamma^{\prime} \in H^{\mu}$. If $\Gamma^{\prime}$ is orthogonal to none of the $\Delta^{\prime}$, then the corresponding entry of $K^{\mu} w$ is clearly zero. Otherwise, for fixed $\Delta$ such that $\Gamma^{\prime} \perp \Delta^{\prime}$, each row of $\Gamma^{\prime}$ has exactly one entry from the first column of $\Delta^{\prime}$, since the length of this first column is equal to the number of rows of $\Gamma^{\prime}$ and by orthogonality no row of $\Gamma^{\prime}$ can contain two elements from any column of $\Delta^{\prime}$. Therefore we may remove the leftmost column from $\Delta^{\prime}$ and the corresponding elements from the rows of $\Gamma^{\prime}$; the resulting tabloids will have shape $\lambda$, and $\Delta^{\prime}$ will be reduced to $\Delta$. Furthermore, the elements removed from $\Gamma^{\prime}$ are precisely the integers $b+1, \ldots, c$, so the resulting $\Gamma \in H^{\lambda}$ is independent of the choice of $\Delta$. Therefore $\Gamma^{\prime} \perp \Delta^{\prime}$ if and only if $\Gamma \perp \Delta$. But then the entry of $K^{\mu} w$ corresponding to $\Gamma^{\prime}$ is $\sum_{\Delta^{\prime} \in V^{\mu}} K_{\Gamma^{\prime}, \Delta^{\prime}}^{\mu} w_{\Delta^{\prime}}=\sum_{\Delta \in V^{\lambda}} K_{\Gamma, \Delta}^{\lambda} v_{\Delta}=0$, and so we conclude that $K^{\mu} w=0$ as desired.

Calling a shape $\lambda$ rank-maximal precisely when $K^{\lambda}$ has maximal rank, we see that Lemma 2.1 provides an easy way to construct shapes which are not rank-maximal. For if we can extend a given $\lambda$ with associated nonzero $v \in \operatorname{ker}\left(K^{\lambda}\right)$ to a shape $\mu$ such that $\left|H^{\mu}\right| \geq\left|V^{\mu}\right|$, then the existence of a nonzero $w \in \operatorname{ker}\left(K^{\mu}\right)$ tells us that $\operatorname{rank}\left(K^{\mu}\right)<\left|V^{\mu}\right|$ and hence that $\mu$ is not rank-maximal.

For example, this implies Black and List's theorem mentioned above, given their construction of the maps $\phi^{m, n}$.

Corollary 2.2. Given integers $n, m$, and $r$ such that $n \geq m>1$ and $m \geq r \geq 1$, if $\phi^{m, n}$ is injective, then $\phi^{r, n}$ is injective as well.

Proof. Suppose that $K^{n \times r}$ does not have full rank. It is easy to show that $K^{n \times m}$ has at least as many rows as columns, and an $n \times m$ rectangle extends an $n \times r$ rectangle. Hence by Lemma 2.1 there must be a nonzero $v \in \operatorname{ker}\left(K^{n \times m}\right)$, meaning that $K^{n \times m}$ does not have full rank either. Equivalently, if $K^{n \times m}$ has full rank, then so does $K^{n \times r}$, and so if $\phi^{m, n}$ is injective then $\phi^{r, n}$ is injective as well.

We now wish to find shapes with more row tabloids than column tabloids to which we can apply Lemma 2.1. Consider a shape $\lambda=\left(\lambda_{1}, \lambda_{2}, \ldots, \lambda_{r}\right)$. For $j \geq 1$, let $m_{j}$ be the 
number of $i$ such that $\lambda_{i}=j$. It is easy to show that the number of row tabloids in $H^{\lambda}$ is given by

$$
\left|H^{\lambda}\right|=\frac{|\lambda| !}{\left(\prod_{i=1}^{r} \lambda_{i} !\right) \cdot\left(\prod_{j} m_{j} !\right)}
$$

or equivalently, $\left|H^{\lambda}\right|=\left(\begin{array}{c}|\lambda| \\ \lambda_{1}, \ldots, \lambda_{r}\end{array}\right)\left(\prod m_{j} !\right)^{-1}$. This is true because the first factor is the number of ways to assign $\lambda_{1}$ numbers to the first row, $\lambda_{2}$ of the remaining numbers to the second row, and so on, and the $\left(\prod m_{j} !\right)^{-1}$ factor compensates for the fact that the order of the rows of a given size within a row tabloid does not matter.

If we extend $\lambda$ by a single column of length $l \geq r$ to get a new shape $\mu$, then it follows from this formula that $\left|H^{\mu}\right|=\frac{(|\lambda|+1) \cdots(|\lambda|+l)}{\prod_{i}\left(\lambda_{i}+1\right) \cdot(l-r) !}\left|\bar{H}^{\lambda}\right|$, where the $(l-r)$ ! term reflects the number of newly created rows of size 1 . We may construct column tabloids with shape $\mu$ by choosing $l$ numbers to place in the first column and then filling the rest of $\mu$ (i.e. $\lambda$ ) in any possible way. Constructing $\mu$ from $\lambda$ changes the number of columns of length $l$ from $\lambda_{l}$ to $\lambda_{l}+1$ (where $\lambda_{l}=0$ if $l>r$ ), so $\left|V^{\mu}\right|=\frac{1}{\lambda_{l}+1}\left(\begin{array}{c}|\lambda|+l \\ l\end{array}\right)\left|V^{\lambda}\right|$. Combining these equations yields

$$
\frac{\left|H^{\mu}\right|}{\left|V^{\mu}\right|}=\left(\frac{l(l-1) \cdots(l-r+1) \cdot\left(\lambda_{l}+1\right)}{\prod\left(\lambda_{i}+1\right)}\right) \frac{\left|H^{\lambda}\right|}{\left|V^{\lambda}\right|}
$$

and so for sufficiently large $l$ we will have $\frac{\left|H^{\mu}\right|}{\left|V^{\mu}\right|} \geq 1$, or $\left|H^{\mu}\right| \geq\left|V^{\mu}\right|$.

Theorem 2.3. Given any shape $\lambda$, there exists a shape $\mu$ constructed by adding a single row to the top of $\lambda$, a single column to the left of $\lambda$, or both, such that $\mu$ is not rankmaximal.

Proof. If $\left|V^{\lambda}\right|>\left|H^{\lambda}\right|$, then the columns of $K^{\lambda}$ must be linearly dependent, so there exists a nonzero $v$ such that $K^{\lambda} v=0$. Extend $\lambda$ by prepending a sufficiently long column as described above, so that the new shape $\mu$ satisfies $\left|H^{\mu}\right| \geq\left|V^{\mu}\right|$. Then by Lemma 2.1 there exists a nonzero vector $w$ satisfying $K^{\mu} w=0$, and thus $K^{\mu}$ cannot have maximal rank.

If $\left|V^{\lambda}\right|<\left|H^{\lambda}\right|$, then since $K^{\lambda^{\prime}}=\left(K^{\lambda}\right)^{\top}$ we have $\left|V^{\lambda^{\prime}}\right|>\left|H^{\lambda^{\prime}}\right|$. If we add a sufficiently long column to $\lambda^{\prime}$ to get a shape $\mu^{\prime}$ which is not rank-maximal, then $\mu$ is not rank-maximal either. But then $\mu$ is the desired shape, since it consists of a single row added on top of $\lambda$.

Otherwise, we must have $\left|V^{\lambda}\right|=\left|H^{\lambda}\right|$. If we add a sufficiently long column to $\lambda$, the resulting shape $\nu$ will satisfy $\left|V^{\nu}\right|<\left|H^{\nu}\right|$, and so we can add a long row on top of $\nu$ to get a shape $\mu$ which is not rank-maximal.

In other words, we have shown that any shape can be placed inside a large enough "hook" (possibly degenerate) to get a shape which is not rank-maximal. This shows that Conjecture 1.1 fails over a large class of shapes. Furthermore, it is easy to see that if $\lambda$ is any symmetric shape, i.e. if $\lambda=\lambda^{\prime}$, then we may choose the hook to be symmetric in the proof of Theorem 2.3, since the resulting shape $\mu$ will satisfy $\left|H^{\mu}\right|=\left|V^{\mu}\right|$. But 

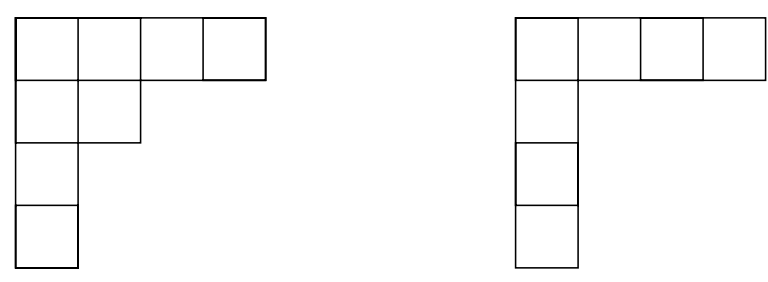

Figure 2: Reinforced hooks such as $(4,2,1,1)$ are not rank-maximal, but ordinary hooks such as $(4,1,1,1)$ are.

symmetric shapes satisfy $\mu \geq \mu^{\prime}$ in dominance order, so we get infinitely many shapes $\mu$ satisfying the hypothesis of Stanley's conjecture for which the rows of $K^{\mu}$ are not linearly independent and thus $\mu$ is not rank-maximal.

Following the proof of Theorem 2.3 with the shape $\lambda=(1)$, for example, we see that the "reinforced hooks" ( $m, 2,1,1, \ldots, 1)$ with $n$ rows, as shown in Figure 2, are not rankmaximal when $m, n \geq 4$. Removing a single box from the second row, however, yields ordinary hooks, which are known to be rank-maximal [5].

Although Theorem 2.3 allows us to construct many counterexamples to Conjecture 1.1, it is unknown how many shapes which are not rank-maximal can be constructed in this way. The author wrote a $\mathrm{C}$ program to generate $K^{\lambda}$ for any shape $\lambda$, and used this together with PARI/GP to determine which $K^{\lambda}$ do not have maximum rank for all shapes $\lambda$ with at most 10 boxes except (due to memory constraints) the shape $(4,3,2,1)$. Among these shapes, the only ones which failed to be rank-maximal were $(4,2,1,1),(5,2,1,1)$, $(5,2,2,1),(5,2,1,1,1),(6,2,1,1),(4,3,3)$, and their transposes. Only the last of these was not explained by Theorem 2.3, which also does not account for the larger known counterexample $(5,5,5,5,5)$ (see [2]).

\section{Minimizing the rank of $K^{\lambda}$}

A natural question at this point is to what extent a given matrix $K^{\lambda}$ can fail to have full rank. In other words, how small can the ratio $\frac{\operatorname{rank}\left(K^{\lambda}\right)}{\min \left(\left|H^{\lambda}\right|,\left|V^{\lambda}\right|\right)}$ be? (Note that this ratio is 1 if and only if $\lambda$ is rank-maximal.) In order to determine this, we strengthen Lemma 2.1 as follows:

Lemma 3.1. Suppose $\operatorname{ker}\left(K^{\lambda}\right)$ has dimension $d$, and that $\lambda$ has $r$ rows. If $\mu$ extends $\lambda$ in such a way that every column of $\mu-\lambda$ has more than $r$ elements, then

$$
\operatorname{dim}\left(\operatorname{ker}\left(K^{\mu}\right)\right) \geq d\left(\begin{array}{l}
|\mu| \\
|\lambda|
\end{array}\right) \cdot\left|V^{\mu-\lambda}\right|
$$

Proof. We may decompose $\mathbb{Q}^{\left|V^{\mu}\right|}$ into a direct sum of $\left(\begin{array}{l}|\mu| \\ |\lambda|\end{array}\right)\left|V^{\mu-\lambda}\right|$ subspaces $W_{i}$, each of dimension $\left|V^{\lambda}\right|$, as follows: in creating a column tabloid of shape $\mu$, there are $\left(\begin{array}{l}|\mu| \\ |\lambda|\end{array} \mid\right) \mid V^{\mu-\lambda \mid}$ ways to pick the $|\mu|-|\lambda|$ numbers which will fill the columns of $\mu-\lambda$ and then assign 
them to columns in vertically inequivalent ways. Fix such an assignment, and for all column tabloids $\Delta_{j}$ which match this assignment, let $v_{\Delta_{j}}$ be the vector whose only nonzero coordinate is a 1 in the entry corresponding to $\Delta_{j}$. The subspace $W_{i}$ associated with the chosen assignment is then defined as the span of these $v_{\Delta_{j}}$. These subspaces intersect only at 0 , and hence we have a direct sum, because the columns of $\mu-\lambda$ have more than $r$ elements each; this allows us to identify a unique filling of $\mu-\lambda$ with each column tabloid because the columns of $\mu-\lambda$ are longer than the columns of $\lambda$ and hence they cannot be exchanged.

We now claim that $\operatorname{dim}\left(\operatorname{ker}\left(K^{\mu}\right) \cap W_{i}\right) \geq d$ for all $i$. Let $\left\{v_{1}, \ldots, v_{d}\right\}$ be a basis of $\operatorname{ker}\left(K^{\lambda}\right)$. As in the proof of Lemma 2.1, it is clear that we may construct from these a set of $d$ linearly independent vectors $w_{1}, w_{2}, \ldots, w_{d} \in \operatorname{ker}\left(K^{\mu}\right)$ which all lie in a particular subspace $W_{1}$. By symmetry, then, we may similarly find $d$ linearly independent vectors in any $\operatorname{ker}\left(K^{\mu}\right) \cap W_{i}$. Hence $\operatorname{dim}\left(\operatorname{ker}\left(K^{\mu}\right)\right) \geq d \cdot\left(\begin{array}{l}|\mu| \\ |\lambda|\end{array}\right)\left|V^{\mu-\lambda}\right|$, as desired.

Consider the symmetric reinforced hooks $\lambda=(m, 2,1, \ldots, 1)$ with $m$ rows, which are clearly counterexamples to both Conjecture 1.1 and Stanley's conjecture. Then $\left|H^{\lambda}\right|=$ $\left|V^{\lambda}\right|=\frac{(2 m) !}{m ! 2 !(m-2) !}=\left(\begin{array}{c}2 m \\ m\end{array}\right)\left(\begin{array}{c}m \\ 2\end{array}\right)$ by Equation 1. These shapes extend the hooks $\nu=(m-$ $1,1)$ by a single column of length $m$. Since $\left|H^{\nu}\right|=m$ and $\left|V^{\nu}\right|=\left(\begin{array}{c}m \\ 2\end{array}\right)$, we know that $\operatorname{dim}\left(\operatorname{ker}\left(K^{\nu}\right)\right) \geq\left(\begin{array}{c}m \\ 2\end{array}\right)-m$, and in fact we have equality since hooks are rank-maximal. By Lemma 3.1, we get $\operatorname{dim}\left(\operatorname{ker}\left(K^{\lambda}\right)\right) \geq\left(\left(\begin{array}{c}m \\ 2\end{array}\right)-m\right)\left(\begin{array}{c}2 m \\ m\end{array}\right)$, and so $\operatorname{rank}\left(K^{\lambda}\right)=\left(\begin{array}{c}2 m \\ m\end{array}\right)\left(\begin{array}{c}m \\ 2\end{array}\right)-$ $\operatorname{dim}\left(\operatorname{ker}\left(K^{\lambda}\right)\right) \leq m\left(\begin{array}{c}2 m \\ m\end{array}\right)$. It follows that

$$
\frac{\operatorname{rank}\left(K^{\lambda}\right)}{\min \left(\left|H^{\lambda}\right|,\left|V^{\lambda}\right|\right)} \leq \frac{m\left(\begin{array}{c}
2 m \\
m
\end{array}\right)}{\left(\begin{array}{c}
2 m \\
m
\end{array}\right)\left(\begin{array}{c}
m \\
2
\end{array}\right)}=\frac{2}{m-1},
$$

which goes to zero as $m \rightarrow \infty$. We have therefore shown the following.

Proposition 3.2. The ratio of $\operatorname{rank}\left(K^{\lambda}\right)$ to the number of rows or columns (whichever is smaller) of $K^{\lambda}$ can be arbitrarily close to zero.

\section{Rank-maximality of squares}

We now consider the rank-maximality of squares, since we are especially interested in rectangles for the application to Foulkes' conjecture and squares are in some sense the simplest of these shapes; in particular, $K^{n \times n}$ is a square matrix, and it is known that $K^{5 \times 5}$ does not have maximum rank while nothing is known for similarly-sized rectangles. It seems likely that $n \times n$ squares are not rank-maximal for $n \geq 5$, so we will consider an approach toward a proof of this conjecture.

Supposing that an $n \times n$ square is not rank-maximal, we will find a lower bound on the size of $\operatorname{ker}\left(K^{n \times(n+1)}\right)$. If this bound is large enough to show that $n \times(n+1)$ rectangles, and hence $(n+1) \times n$ rectangles, are not rank-maximal, we note that $(n+1) \times(n+1)$ squares extend $(n+1) \times n$ rectangles and then apply Lemma 2.1. We cannot apply Lemma 3.1 directly, since the column added in the extension to an $n \times(n+1)$ rectangle is no 
longer than the columns of the original square, and so we require a new way to estimate the dimension of $\operatorname{ker}\left(K^{n \times(n+1)}\right)$.

Lemma 4.1. Let $d=\operatorname{dim}\left(\operatorname{ker}\left(K^{n \times n}\right)\right)$. Then $\operatorname{dim}\left(\operatorname{ker}\left(K^{n \times(n+1)}\right)\right) \geq\left(\begin{array}{c}n^{2}+n-1 \\ n-1\end{array}\right) d$.

Proof. As in the proof of Lemma 3.1, we may fill the leftmost column of the $n \times(n+1)$ rectangle with any $n$ of the numbers $1, \ldots, n^{2}+n$ and then use the remaining numbers to construct elements of $\operatorname{ker}\left(K^{n \times(n+1)}\right)$ from elements of $\operatorname{ker}\left(K^{n \times n}\right)$. The $\left(\begin{array}{c}n^{2}+n \\ n\end{array}\right)$ resulting $d$-dimensional subspaces of $\operatorname{ker}\left(K^{n \times(n+1)}\right)$ may intersect in such a way that a linear dependence is created. However, the $\left(\begin{array}{c}n^{2}+n-1 \\ n-1\end{array}\right)$ subspaces which we identify with the tabloids containing the number $n^{2}+n$ in the leftmost column will not. The reason for this is that if we decompose $\mathbb{Q}^{\left|V^{n \times(n+1)}\right|}$ into subspaces $W_{i}$, where similarly to Lemma 3.1 we define each $W_{i}$ to be the span of the vectors $v_{\Delta_{j}}$ over all $\Delta_{j}$ with a fixed column containing the number $n^{2}+n$, then each $d$-dimensional subspace of $\operatorname{ker}\left(K^{n \times(n+1)}\right)$ is contained within a unique $W_{i}$. It follows that $\operatorname{dim}\left(\operatorname{ker}\left(K^{n \times(n+1)}\right)\right) \geq\left(\begin{array}{c}n^{2}+n-1 \\ n-1\end{array}\right) d$, as desired.

The matrix $K^{n \times(n+1)}$ has $\frac{\left(n^{2}+n\right) !}{(n+1) ! n \cdot n !}$ rows and $\frac{\left(n^{2}+n\right) !}{n !^{n+1} \cdot(n+1) !}$ columns, and it can be shown that the latter of these numbers is greater, so $n \times(n+1)$ rectangles are not rank-maximal if and only if $\operatorname{dim}\left(\operatorname{ker}\left(K^{n \times(n+1)}\right)\right)$ exceeds the number of columns minus the number of rows. Using the estimate of Lemma 4.1, then, it suffices to know that

$$
\left(\begin{array}{c}
n^{2}+n-1 \\
n-1
\end{array}\right) d>\frac{\left(n^{2}+n\right) !}{n !^{n+1}}\left(\frac{1}{(n+1) !}-\frac{1}{(n+1)^{n}}\right)
$$

or, simplifying, that

$$
\begin{aligned}
d & >\frac{\left(n^{2}+n\right) \cdot(n-1) ! \cdot\left(n^{2}\right) !}{n !^{n+1}}\left(\frac{1}{(n+1) !}-\frac{1}{(n+1)^{n}}\right) \\
& =\left(\frac{\left(n^{2}\right) !}{n !^{n+1}}\right)\left(1-\frac{(n+1) !}{(n+1)^{n}}\right) .
\end{aligned}
$$

The factor on the left is the number of rows (or columns) of $K^{n \times n}$, and as explained above we know via Lemma 2.1 that the matrix $K^{(n+1) \times(n+1)}$ does not have full rank if $K^{n \times(n+1)}$ does not, so we conclude the following.

Proposition 4.2. Suppose that an $n \times n$ square is not rank-maximal. If $\frac{\operatorname{rank}\left(K^{n \times n}\right)}{\left|H^{n \times n}\right|}<$ $\frac{n !}{(n+1)^{n-1}}$, then the $(n+1) \times(n+1)$ square is not rank-maximal either.

For example, we know that $K^{5 \times 5}$ does not have full rank. If $\frac{\operatorname{rank}\left(K^{5 \times 5}\right)}{\left|H^{5 \times 5}\right|}<\frac{5}{54}$, which is currently unknown since we do not even have an estimate of $\operatorname{rank}\left(K^{5 \times 5}\right)$, then $K^{6 \times 6}$ does not have full rank either. This method may suffice in general to prove that many rectangles are not rank-maximal - in particular, it would already show this for $n \times(n+1)$ rectangles - and therefore that the method of Black and List cannot resolve many cases of Foulkes' conjecture. We leave it as an open problem to strengthen the bound of Proposition 4.2 if possible. 


\section{The kernel of $K^{\lambda}$}

Let $\lambda$ be any shape for which $\operatorname{ker}\left(K^{\lambda}\right)$ has positive dimension; this is the case when $\lambda$ is not rank-maximal, but it is also true of rank-maximal shapes satisfying $\left|V^{\lambda}\right|>\left|H^{\lambda}\right|$. The following statement seems to be generally true.

Conjecture 5.1. If $\operatorname{ker}\left(K^{\lambda}\right)$ has positive dimension, then there exists a nonzero vector $v \in \operatorname{ker}\left(K^{\lambda}\right)$ all of whose entries are 0,1 , or -1 . Equivalently, there exist two disjoint subsets $A, B \subset V^{\lambda}$ such that $|\{\Delta \in A: \Gamma \perp \Delta\}|=|\{\Delta \in B: \Gamma \perp \Delta\}|$ for all $\Gamma \in H^{\lambda}$.

The equivalence follows from letting $A$ consist of the $\Delta$ corresponding to positive entries and likewise assigning the $\Delta$ with negative entries to $B$. Using the first of these conditions, we immediately see by the proof of Lemma 2.1 that if Conjecture 5.1 is true for some $\lambda$, then it is also true for any shape $\mu$ which extends $\lambda$.

We also propose a very strong version of this conjecture, for which there is currently much less evidence:

Conjecture 5.2. There is a basis of $\operatorname{ker}\left(K^{\lambda}\right)$ consisting of vectors whose entries are all 0,1 , or -1 .

This can be easily checked for some simple cases, such as $\lambda=(3,1)$ (the smallest shape for which $\operatorname{dim}\left(\operatorname{ker}\left(K^{\lambda}\right)\right)>0$ ), but it is not yet known in any more general sense. However, the weaker version can be proved for a large class of shapes.

Theorem 5.3. Conjecture 5.1 is true for any $m \times n$ rectangle, where $n>m>1$.

Proof. We give an explicit construction of the sets $A$ and $B$ : Fix a way to assign the numbers $n+1, \ldots, m n$ to an $m \times n$ rectangular diagram so that every column contains exactly $m-1$ numbers. Then let $A$ consist of $\frac{n !}{2}$ column tabloids, where each one has $n+1, \ldots, m n$ assigned in the fixed way, and $1, \ldots, n$ are placed one per column with the order determined by an arbitrary even permutation. $B$ is constructed identically, except that the $\frac{n !}{2}$ odd permutations of $1, \ldots, n$ are used.

To show that these sets have the desired property, fix $\Gamma \in H^{\lambda}$ and let $A^{\prime}$ and $B^{\prime}$ be the subsets of $A$ and $B$ whose members are orthogonal to $\Gamma$. Since $n>m$, some row of $\Gamma$ must contain at least two of the numbers $1, \ldots, n$, so assume without loss of generality that 1 and 2 are in the same row. Then the map $\sigma: V^{\lambda} \rightarrow V^{\lambda}$ which swaps 1 and 2 is its own inverse (in particular, it is injective), and it preserves orthogonality to $\Gamma$. But $\sigma\left(A^{\prime}\right) \subset B^{\prime}$, since applying the transposition $\left(\begin{array}{ll}1 & 2\end{array}\right)$ sends even permutations to odd ones, and likewise $\sigma\left(B^{\prime}\right) \subset A^{\prime}$. Therefore $\sigma$ restricts to a bijection $\sigma^{\prime}: A^{\prime} \rightarrow B^{\prime}$, and in particular $\left|A^{\prime}\right|=\left|B^{\prime}\right|$ as desired.

This type of construction seems useful because it might provide a better understanding of precisely why certain shapes are not rank-maximal. In particular, such a description of any member of $\operatorname{ker}\left(K^{5 \times 5}\right)$ might be easily extensible to a proof that no $n \times n$ square is rank-maximal when $n \geq 5$, or even more generally that $m \times n$ rectangles are not rankmaximal for $m, n \geq 5$. Neither of these statements is known to be true or false for any cases except for $5 \times 5$ squares, which are not rank-maximal, but it seems likely that both statements are true. 


\section{Open questions}

In addition to Conjectures 5.1 and 5.2, the following questions are still open and of particular interest because of their relation to Foulkes' conjecture:

1. For which integers $m$ and $n$ does $K^{m \times n}$ have full rank? In particular, are any $m \times n$ rectangles rank-maximal when $m, n \geq 5$ ? Pylyavskyy proved in [4] that $2 \times n$ rectangles are rank-maximal, but the $3 \times n$ case of this remains open even though Foulkes' conjecture is known to be true for $m=2,3,4$.

2. Can the bound of Proposition 4.2 be improved? Alternatively, can we bound $\operatorname{rank}\left(K^{5 \times 5}\right)$ from above well enough to show by Proposition 4.2 that $5 \times 6$ rectangles and $6 \times 6$ squares are not rank-maximal?

3. Describe any nonzero element of $\operatorname{ker}\left(K^{5 \times 5}\right)$ satisfying Conjecture 5.1. It is known that $K^{n \times n}$ has full rank for $n \leq 4$ [2], so why is there nothing analogous to this element for $K^{n \times n}$ with $n$ small?

\section{Acknowledgements}

This research was performed under the supervision of Joseph A. Gallian in his REU program at the University of Minnesota Duluth. The program was supported by NSF grant DMS 0447070 and NSA grant H98230-04-1-0050. The author would like to thank David Moulton, Phil Matchett Wood, David Arthur, and Joseph A. Gallian for providing helpful advice and comments on several versions of this paper.

\section{References}

[1] S. C. Black and R.J. List, A note on plethysm, European Journal of Combinatorics 10 (1989), no. 1, 111-112.

[2] J. Müller and M. Neunhöffer, Some computations regarding Foulkes' conjecture, http://www.math.rwth-aachen.de/ Max. Neunhoeffer/Publications/.

[3] PARI/GP, version 2.1.5, Bordeaux, 2005, http://pari.math.u-bordeaux.fr/.

[4] P. Pylyavskyy, On plethysm conjectures of Stanley and Foulkes: the $2 \times n$ case, Electronic Journal of Combinatorics 11(2) (2004), \#R8.

[5] R. Stanley, Positivity problems and conjectures in algebraic combinatorics, Mathematics: Frontiers and Perspectives, American Mathematical Society, Providence, RI, 2000, pp. 295-319. 